\title{
DENSE ORBITS OF RATIONALS
}

\author{
MICHAEL D. BOSHERNITZAN
}

(Communicated by Andreas R. Blass)

\begin{abstract}
Let $\mathbb{Q}$ denote the rational points of the interval $K=[0,1)$. We construct a one-to-one piecewise linear map $\phi: K \rightarrow K$ which has the following properties:

(1) for any $x \in K, \phi(x) \in \mathbb{Q}$ if and only if $x \in \mathbb{Q}$;

(2) all the orbits $O(x)=\left\{\phi^{i}(x) \mid i \geq 0\right\}, x \in K$, are dense in $K$;

(3) $\phi$ is an automorphism of the unit circle $K=[0,1)=\mathbb{R} / \mathbb{Z}$.

This example is motivated by a question of Friedman who was interested, because of an application to logic (Dynamic Recursion Theory), in an example of a piecewise polynomial map $\phi: K \rightarrow K$ having an orbit $O(k)$ that is dense in $K$ and lies in $\mathbb{Q}$ (for some $k \in K$ ).
\end{abstract}

Let $a, b$ be two real numbers in the open interval $(0,1)$. Define $\phi: K \rightarrow$ $K, K=[0,1)$, by

$$
\phi_{a, b}(x)=\phi(x)= \begin{cases}b+\frac{(1-b) x}{a} & \text { if } 0 \leq x<a, \\ \frac{b}{1-a}(x-a) & \text { if } a \leq x<1 .\end{cases}
$$

(See Figure 1 on the next page for a graph of $\phi$.)

We observe that if $b+a=1$, then $\phi_{a, b}=T_{b}$ where $T_{b}$ is the $b$-rotation $\left(T_{b}(x)=x+b \bmod 1\right)$. It is well known that if $b$ is irrational then $T_{b}$ is uniquely ergodic and, in particular, each orbit is dense in $K$.

In what follows we assume that $b+a<1$. Then $k_{1}=(1-b) / a>1$ and $k_{2}=b /(1-a)<1$ are the slopes of two linear components of the graph of $\phi_{a, b}(x)$. Let $k=k_{1} / k_{2}>1$ and define $\pi: K \rightarrow K$ by

$$
\pi(x)=\frac{\ln [1+(k-1) x]}{\ln k}, \quad \text { for } x \in K=[0,1) .
$$

One verifies that $\pi$ is an automorphism of $K$ and that

$$
\phi_{a, b}(x)=\pi^{-1}\left(T_{c}(\pi(x))\right)=\left(\pi^{-1} \circ T_{c} \circ \pi\right)(x)
$$

where $c=\ln k_{1} / \ln k=\ln k_{1} /\left(\ln k_{1}-\ln k_{2}\right)$ and $T_{c}$ denotes the $c$-rotation $\phi_{1-c, c}$ of the unit circle $K=[0,1)=\mathbb{R} / \mathbb{Z}$. Thus $\phi_{a, b}$ and $T_{c}$ are conjugate

Received by the editors August 5, 1991.

1991 Mathematics Subject Classification. Primary 54H20; Secondary 28E15.

Research supported by research grant NSF-DMS-9003450. 


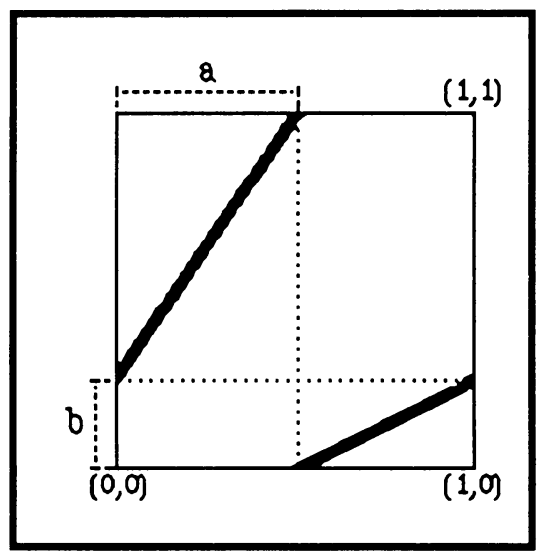

FIGURE 1. Graph of $\phi$.

(by $\pi$ ) and, therefore, the rotation number of the circle automorphism $\phi_{a, b}$ is $c$.

Thus in order for $\phi_{a, b}(x)=\phi(x)$ to satisfy the conditions (1), (2), and (3) (see the abstract), we need to have $a, b \in \mathbb{Q}$, but $c \notin \mathbb{Q}$. Note that

$$
c \notin \mathbb{Q} \Leftrightarrow k_{1}, k_{2} \text { are multiplicatively independent }
$$

(which means that $\ln k_{1}$ and $\ln k_{2}$ are linearly independent over $\mathbb{Q}$ ). One can take (e.g.) $a=\frac{2}{5}$ and $b=\frac{1}{5}$. Then $k_{1}=2, k_{2}=\frac{1}{3}$ are multiplicatively independent and the map $\phi: K \rightarrow K$ defined by

$$
\phi(x)= \begin{cases}\frac{1}{5}+2 x & \text { if } 0 \leq x<\frac{2}{5} \\ \frac{1}{3}\left(x-\frac{2}{5}\right) & \text { if } \frac{2}{5} \leq x<1\end{cases}
$$

is conjugate to the irrational rotation $T_{c}$ with $c=\ln 2 / \ln 6$. Thus the above map $(*)$ satisfies all the conditions listed in the abstract. In particular, the rotation number of the circle automorphism $\phi$ is $\ln 2 / \ln 6 \notin \mathbb{Q}$.

Remark. Note that the maps $\phi_{a, b} \circ T_{c}$ have been considered by Michael Herman [H, §6.3]. For special choices of $a, b$, and $c$, it was shown that the unique invariant probability measure on $K$ (under $\phi_{a, b} \circ T_{c}$ ) cannot be absolutely continuous. The author first observed (using an indirect, long argument) that the rotation number of $\phi_{a, b}$ is $c=\ln k_{1} /\left(\ln k_{1}-\ln k_{2}\right)$ and that (if $\left.c \notin \mathbb{Q}\right)$ the unique invariant probability measure $\eta$ on $K$ (under $\phi_{a, b}$ ) is absolutely continuous relative to the Lebesgue measure $\lambda$, with the Radon-Nikodym derivative $d \eta / d \lambda$ lying inbetween two positive constants. This led the author to search for a nice automorphism, $\pi$, conjugating $\phi_{a, b}$ with $T_{c}$ to be accountable for this phenomenon.

\section{ACKNOWLEDGMENT}

The author is grateful to Harvey Friedman and Liming Ren [R] for explaining to him that the example constructed in this note has an application to the Dynamic Recursion Theory (studying the analogy between Turing Machine and the action of piecewise affine functions) they are developing. 


\section{REFERENCES}

[H] Michael Herman, Sur la conjugason différentiable des diffeomorphisms du cercle à des rotations, Inst. Hautes Études Sci. Publ. Math. 49 (1979), 5-253.

[R] Liming Ren, personal communication, May 1991.

Department of Mathematics, Rice University, Houston, Texas 77251

E-mail address: michael@rice.edu 